\title{
Simulation Study on Various Double Pendulum Configurations
}

\author{
Milena Sipovac ${ }^{1 *}$, Stefanie Winkler ${ }^{1}$, Andreas Körner ${ }^{1}$ \\ ${ }^{1}$ Institute of Analysis and Scientific Computing, Vienna University of Technology, Wiedner Haupstraße 8-10, \\ 1040 Vienna, Austria; ${ }^{*}$ milena.sipovac@tuwien.ac.at
}

\begin{abstract}
This paper is offers an introductory overview of some methods used for first principle modeling of different structures for the double pendulum. The mathematical models are based on the Euler-Lagrange equations. The basic simulation study is focused on the planar double pendulum. Chaotic and periodic behavior is investigated, together with an influence of the external force. For initial conditions close to zero a periodic motion is observable. This model is further extended to a simplified model of a church bell with a double clapper. The clapper is modeled as a double pendulum with limitation of movement inside the sides of the bell. Periodicity under different initial conditions is investigated.
\end{abstract}

\section{Motivation}

The pendulum, together with its variations, is a simple mechanical system that is suitable for applying whitebox modeling techniques, since we know a lot about the system and its movement. In this paper, we will also extend the simple pendulum to a double pendulum. The planar double pendulum is a mechanical system that shows chaotic behavior even though it is a simple system, but its simple construction allows us to observe and understand the behavior of the chaotic systems more closely. We are observing a deterministic system, which means it is defined by its initial conditions, there are no random elements involved, yet because of its chaotic nature, it is still not predictable. The definition of chaos used here is the sensitivity to the change in initial condition. In this paper, we concentrate on the distinction between chaotic and periodic behavior of the system and the initial conditions that influence this, as well as on influence of an external force to its behavior.

The second model observed in this paper is a model of a simplified church bell, with a double pendulum as a clapper. Its purpose is a demonstration of the law of conservation of momentum, since the energy is trans- ferred and distributed between the bell sides and the clapper. It is implemented through modeling the sides of the bell as a simple pendulum, and they are acting as a moving constraint to a double pendulum. The possibility of rhythmic motion is investigated by some parameter studies. This is all implemented by using the MATLAB ODE solver ode45 and by incorporating an event function, which would stop the solver each time an impact happens, and continue with new initial conditions based on the law of conservation of momentum and energy.

\section{Introductory example}

As an introduction to the work, we will start with a simple pendulum in two dimensions. This is a pendulum with a point-mass $m$ attached to a massless, rigid rod of the length $l$, and the equations of motion are derived using the Euler-Lagrange formalism. The system is constrained by the fixed length $l$ of the rod, so the points of mass with coordinates $\left(x_{m}, y_{m}\right)$ have to satisfy $x_{m}^{2}+y_{m}^{2}=l^{2}$.

This means the mass point follows a circular path, and we can use polar coordinates. This is applied on all the further models in this paper. The position is therefore uniquely defined by a position angle $\varphi$. With this information we can obtain the Lagrangian and the equation of the motion of this system, with $g$ as the gravity acceleration constant.

$$
\ddot{\varphi}=-\frac{g \sin \varphi}{l}
$$

The motion is then fully described by the equation (1).

\section{The Double Pendulum}

By following the principle described in the introduction, we can derive the equations of motion for the dou- 
ble pendulum. The setup for this model can be seen on a sketch in Figure 1.

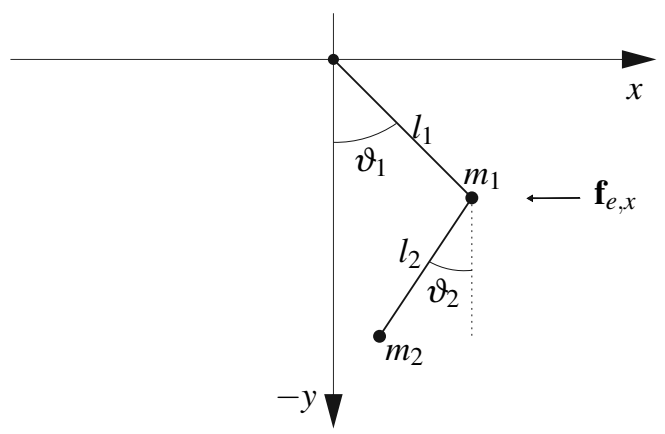

Figure 1: Schematic sketch of a double pendulum.

The positions of the two mass points $m_{1}$ and $m_{2}$ are uniquely described by their position angles $\vartheta_{1}$ and $\vartheta_{2}$, since they are both attached to rigid rods of fixed length $l_{1}$ and $l_{2}$ respectively. The external force is applied on the first mass point $m_{1}$. The motion is described by a system of two non-linear ordinary differential equations of second order.

$$
\begin{aligned}
\ddot{\vartheta}_{1} & =-\frac{g}{l_{1}} \sin \vartheta_{1}-\frac{m_{2}}{m_{1}+m_{2}} \frac{l_{1}}{l_{2}}\left(\cos \left(\vartheta_{1}-\vartheta_{2}\right) \ddot{\vartheta}_{2}\right. \\
& \left.+\sin \left(\vartheta_{1}-\vartheta_{2}\right) \dot{\vartheta}_{2}^{2}\right) \\
\ddot{\vartheta}_{2} & =-\frac{g}{l_{2}} \sin \vartheta_{2}-\frac{l_{1}}{l_{2}} \frac{l_{1}}{l_{2}}\left(\cos \left(\vartheta_{1}-\vartheta_{2}\right) \ddot{\vartheta}_{1}\right. \\
& \left.+\sin \left(\vartheta_{1}-\vartheta_{2}\right) \dot{\vartheta}_{1}^{2}\right)
\end{aligned}
$$

\subsection{Autonomous case}

Before applying any external forces, we model the behavior of the system without the external forces applied. We solve the system (2) by using ode45 from MAT$\mathrm{LAB}$, and investigate different initial conditions.

For the initial conditions less than $\vartheta_{i, 0}={ }_{3}^{\pi}, i=1,2$, a periodic motion is visible, whereas a non-periodic motion is visible for initial conditions larger than $\vartheta_{i, 0}=$ $\frac{\pi}{3}, i=1,2$, see Figure 2 . This can be explained with the small-angle approximations, which lead to linearization of equations in the system (2). These linearized equations have periodic solutions.

In the Figure 3 we can see a demonstration of the chaotic motion, with a small change in initial condition with no initial velocity. This property is better visible in the motion of the second mass point, which is also be seen on the graph.

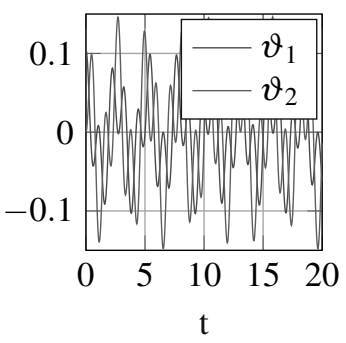

(a)

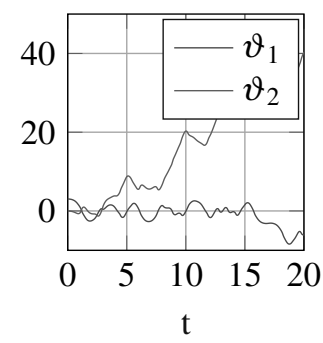

(b)
Figure 2: Motion for initial conditions (a) $\vartheta_{1,0}=\frac{\pi}{30}$ and $\vartheta_{2,0}=0$,

(b) $\vartheta_{1,0}=\frac{29 \pi}{30}$ and $\vartheta_{2,0}=0$.

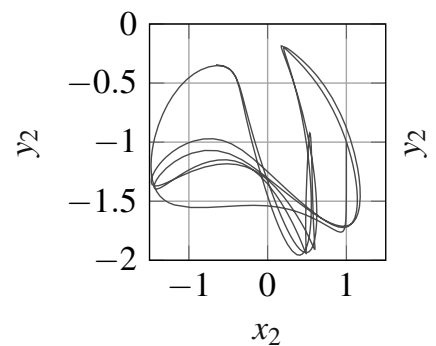

(a)

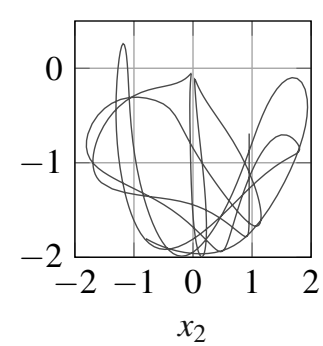

(b)
Figure 3: Motion for initial conditions (a) $\vartheta_{1,0}=\frac{\pi}{30}$ and $\vartheta_{2,0}=0$,

(b) $\vartheta_{1,0}=\frac{29 \pi}{30}$ and $\vartheta_{2,0}=0$.

\subsection{Motion with external Stimulation}

In this subsection we are observing the behavior of the double pendulum when an external force is applied, in the direction of the $x$-axis. Therefore, the external force is given by $\mathbf{f}_{e}=\left(\mathbf{f}_{e, x}, 0,0\right)^{T}$. Since the generalized coordinates are $\left(\vartheta_{1}, \vartheta_{2}\right)$, the external force also has two coordinates, $\mathbf{f}_{\vartheta_{1}}$ and $\mathbf{f}_{\vartheta_{2}}$. With this, we obtain an inhomogeneous equation system, where the inhomogeneities correspond to

$$
\mathbf{f}_{\vartheta_{1}}=\mathbf{f}_{e, x} l_{1} \cos \vartheta_{1}, \quad \mathbf{f}_{\vartheta_{2}}=\mathbf{f}_{e, x} l_{2} \cos \vartheta_{2}
$$

In the Figure 4(a) we can see a trajectory of the motion of the double pendulum with an external force applied, and compared to the Figure 4(b), where no external force is applied, we can observe a limited motion in the first figure, since the external force represents a pull in the positive $x$-direction. 


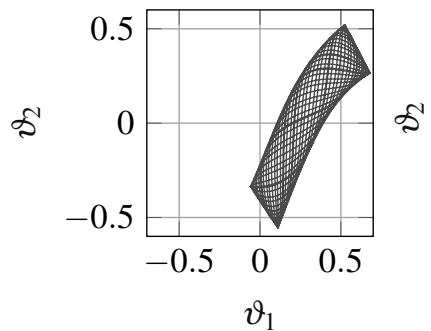

(a)

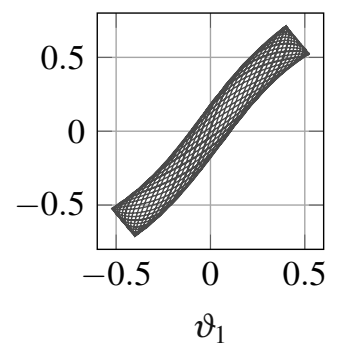

(b)
Figure 4: Trajectories for initial conditions $\vartheta_{1,0}=\frac{\pi}{6}$ and $\vartheta_{2,0}=\frac{\pi}{6}, t \in[0,40]$. (a) External force $\mathbf{f}_{e}=(3,0,0)^{T}$ applied. (b) No external force applied.

\section{Bell With a Double Clapper}

The basic model of a double pendulum is further extended to a simplified bell with a double clapper, as illustrated in Figure 5.

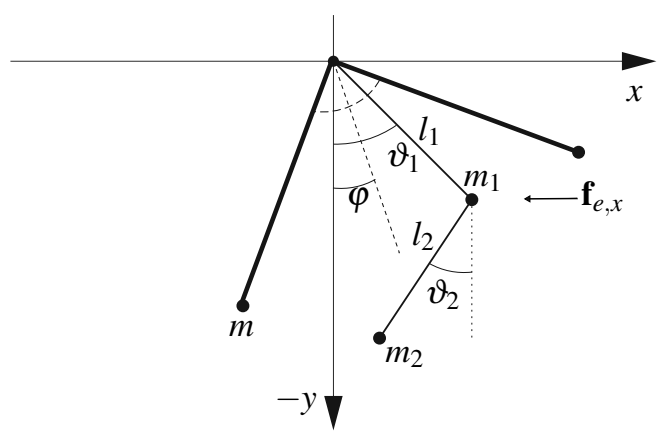

Figure 5: Mechanical setup for the model of a bell.

The outer part of the bell is observed as two coupled simple pendulums with a fixed angle between the rods. The mass of the whole bell is considered concentrated in the mass points $m$ and the rods are considered to be massless. The clapper of the bell is modeled as a double pendulum, with two mass points $m_{1}$ and $m_{2}$. An external force is applied to the mass $m_{1}$. The equations used here correspond to a system with coupled independent equations (1) and (2). The angle $\varphi$ determines the position of the bell sides, and $\vartheta_{1}$ and $\vartheta_{2}$ determine the positions of the respective mass points of the bell clapper, all labeled in Figure 5. Depending on which of the two mass points hits the wall of the bell for $i=1,2$, we are going to use the law of conservation of energy and momentum, to obtain the new conditions after each collision, assumed the system is not damped.

$$
\begin{aligned}
m \dot{\varphi}\left(t^{-}\right)+m_{i} \dot{\vartheta}_{i}\left(t^{-}\right) & =m \dot{\varphi}\left(t^{+}\right)+m_{i} \dot{\vartheta}_{i}\left(t^{+}\right) \\
\frac{1}{2} m \dot{\varphi}\left(t^{-}\right)^{2}+\frac{1}{2} m_{i} \dot{\vartheta}_{i}\left(t^{-}\right)^{2} & =m \frac{1}{2} \dot{\varphi}\left(t^{+}\right)^{2}+\frac{1}{2} m_{i} \dot{\vartheta}_{i}\left(t^{+}\right)^{2}
\end{aligned}
$$

The behavior of the bell is investigated by some parameter studies. Each time the normal distance netween the mass point and the outer part of the bell would equal zero, an event is triggered, which would stop the solver. The mass of the outer part of the bell is held fixed, and significantly greater than the mass of the clapper. The opening angle is held fixed at $\alpha=\frac{\pi}{3}$.

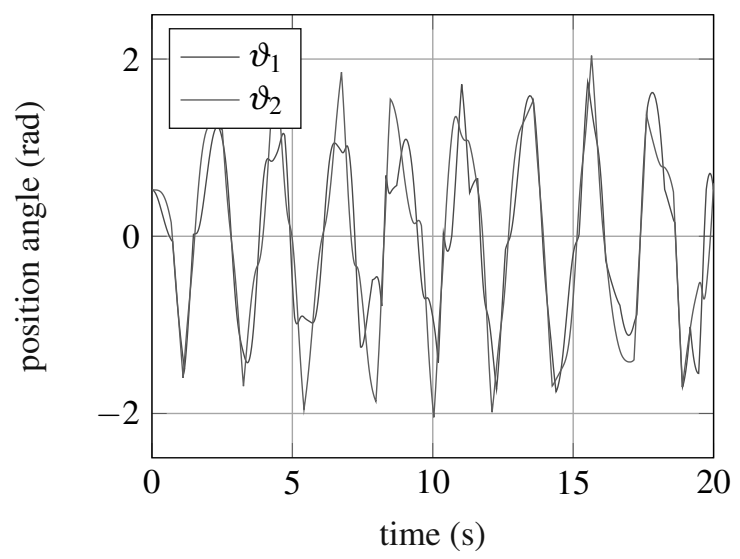

Figure 6: Model of a bell with a double clapper,

$$
\begin{aligned}
& m_{1}=m_{2}=40 \mathrm{~kg}, m=2000 \mathrm{~kg}, l=3 \mathrm{~m}, l_{1}=1 \mathrm{~m}, \\
& l_{2}=1.5 \mathrm{~m}, \text { position angles of the clapper plotted } \\
& \text { over time. }
\end{aligned}
$$

On the Figure 6 we can see a bell with no external stimulation, and the bell is brought to ringing by displacing the bell together with the clapper, and letting it swing rather than pushing with an external influence. The initial conditions are $\varphi_{0}=\frac{\pi}{3}, \vartheta_{1,0}=\frac{\pi}{6}, \vartheta_{2,0}=\frac{\pi}{6}$. The events can be seen on the sharp edges on the plot, where the movement was interrupted by an impact on the side of the bell.

Another possibility of ringing a bell, by giving an initial velocity to the bell, but keeping the position angles of the clapper at zero, i.e. giving the bell a push on the side instead of letting it swing. The initial velocity is $\dot{\varphi}_{0}=1.8 \mathrm{rad} / \mathrm{s}$. The motion induced in this way can be seen on the Figure 7.

The question of the possibility of rhythmic motion can be answered by making a scatter-plot of time differences between consecutive impacts. This can be ob- 


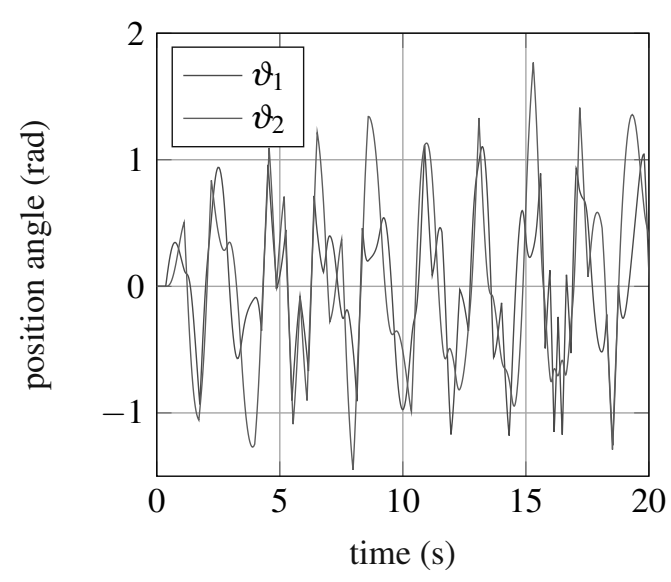

Figure 7: Model of a bell with a double clapper, $m_{1}=m_{2}=40 \mathrm{~kg}, m=2000 \mathrm{~kg}, l=3 \mathrm{~m}, l_{1}=1 \mathrm{~m}$, $l_{2}=1.5 \mathrm{~m}$, position angles of the clapper plotted over time.

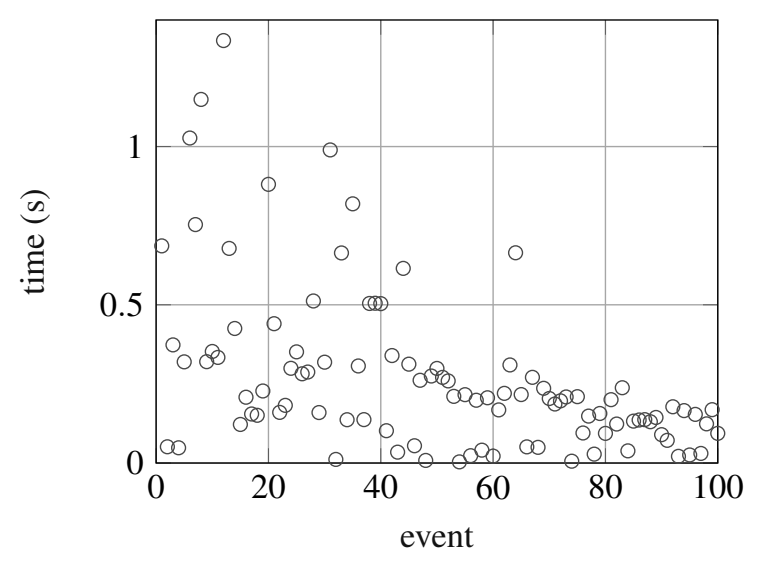

Figure 8: Swinging bell.

served on Figure 8 for the swinging bell, and on Figure 9 for the pushed bell, with initial conditions mentioned above. We can see that the swinging bell, after, and with the pushed bell it does not happen, with the given initial conditions.

\section{Conclusion}

The main idea of this paper was to investigate the behavior of pendulum systems, by modeling them from first principles. We started with a double pendulum and investigated its chaotic behavior. This model could be extended to a three dimensional model, and by using the external force, or damping, different surroundings

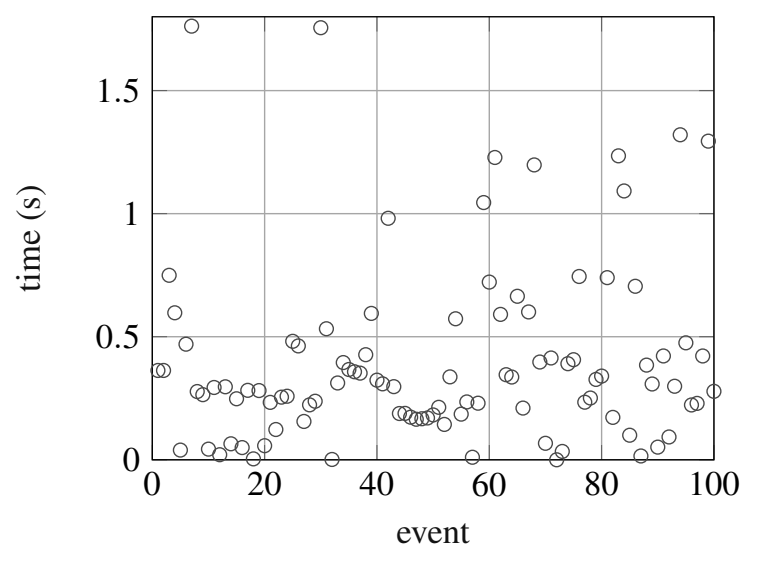

Figure 9: Pushed bell.

could be investigated.

The second model was a simplified model of a bell, where we saw that with a double pendulum as a clapper, periodic motion is not easy to obtain, due to the chaotic behavior of the double pendulum. Taking the material properties of which the bell is made could be an improvement, as well as considering a three dimensional model here as well. Certain parameters were not investigated and this could be included in the future work.

\section{References}

[1] Hasselblatt B., Katok A. Introduction to the Modern Theory of Dynamical Systems. Cambridge University Press, 1995.

[2] Saber N. Elaydi Discrete Chaos. Chapman and Hall, 1999.

[3] Scheck F., Mechanics - From Newton's Laws to Deterministic Chaos. Springer-Verlag Berlin Heidelberg, 2010.

[4] Dudtschenko K., Küpper T., Hosham A.. The dynamics of bells as an impacting system Proceedings of the Institution of Mechanical Engineers, Part C: Journal of Mechanical Engineering Science. 2010; 225(10):2436-2443.

[5] Stachowiak T., Okada T.. A numerical analysis of chaos in the double pendulum. Chaos, Solitons \& Fractals, Elsevier. 2006; 29(2):417-422.

[6] Menghetti G., Rossi B.. An analytical model based on lumped parameters for the dynamic analysis of church bells. Engineering Structures, Elsevier. 2010; 32(10):3363-3376. 